\title{
Motor neurone excitability in back muscles assessed using mechanically evoked reflexes in spinal cord injured patients
}

\author{
A Kuppuswamy, S Theodorou, M Catley, P H Strutton, P H Ellaway, A H McGregor, N J Davey
}

J Neurol Neurosurg Psychiatry 2005;76:1259-1263. doi: 10.1136/jnnp.2004.045021

See end of article for authors' affiliations

Correspondence to:

Dr N J Davey, Department of Movement and Balance, Division of Neuroscience and Psychological Medicine, Imperial College Faculty of Medicine, Charing Cross Campus, St Dunstan's Road, London W6 8RP, UK; n.davey@ imperial.ac.uk

Received 6 May 2004

In revised form

9 November 2004

Accepted

25 November 2004
Objective: The clinical and functional assessment of back muscles in human spinal cord injury (SCI) has received little attention. The aim of this study was to develop a method to assess the level of a thoracic spinal cord lesion based on the reflex activation of back muscles.

Methods: In 11 control subjects and in 12 subjects with clinically complete thoracic SCI (T2-T12), either a spinous process or an erector spinae muscle was prodded to elicit short latency reflexes recorded electromyographically at the spinal level of stimulation. An electromagnetic servo, attached to a blunt probe, applied stimuli at a frequency of $1 \mathrm{~Hz}$ and amplitude of $3 \mathrm{~mm}$. Two trials of 50 mechanical prods were conducted at each site.

Results: Reflexes were evoked in control subjects in $82 \%$ of trials when the spinous process was prodded, and in $80 \%$ of trials when the muscle was prodded. In contrast, reflexes in $\mathrm{SCl}$ subjects could be elicited in 90-100\% of trials two segments either above or below the lesion. Reflex responses in control subjects had a mean (SEM) latency of $5.72(0.53) \mathrm{ms}$ when the spinous process was prodded, and $5.42(0.42) \mathrm{ms}$ when the muscle was prodded. In the $\mathrm{SCl}$ subjects, responses had slightly (but insignificantly) longer latencies both above and below the lesion to either stimulus. The amplitude of reflex responses, expressed as a percentage of the background EMG, was on average 2-3 times larger at the three vertebral levels spanning the lesion in $\mathrm{SCl}$ subjects than at sites above or below the lesion or at any level in control subjects. Conclusion: We propose that the size of these mechanically evoked reflexes may be useful in determining the level of thoracic $\mathrm{SCl}$. Furthermore, the reflexes might provide a valuable tool with which to monitor recovery after an intervention to repair or improve function of a damaged spinal cord.
S pinal cord injury (SCI) can be classified based on the level of lesion and on the completeness (density) of lesion. American Spinal Injury Association (ASIA) assessment scores are widely used to assess the level and density of SCI, but do not provide an accurate picture, especially within the thoracic cord. In addition, the ASIA assessment relies only on the sensory scores to classify thoracic lesions and does not attempt to assess muscles of the trunk. Quantification of treatment outcome is an important feature of assessment scores, and ASIA assessment scores lack the sensitivity to monitor treatment outcome accurately. ${ }^{2}$ The need for accurate classification and prediction of outcome of spinal cord injury has become increasingly important ${ }^{3}{ }^{4}$ with the advent of new cellular and molecular interventional therapies in pre-clinical studies. ${ }^{56}$ Recovery from spinal cord injury is influenced by a number of factors, and it is difficult to demonstrate the effect of a single treatment. ${ }^{7}$ To identify the various components of recovery, specific neurophysiological tests are required. A number of such neurophysiological tools for assessing spinal cord injury have been assessed recently. ${ }^{8-14}$ In this paper, we investigate the reflex activation of back muscles as the basis for a neurophysiological test for assessment of level of a thoracic lesion in chronic SCI patients that may also be a potential indicator of treatment outcome. The work relates to a previous study, ${ }^{15}$ in which it was speculated that the amplitude and latency of short latency reflexes elicited in trunk muscles could prove to be a useful diagnostic tool for evaluating nerve root compressions.

Spinal reflexes are subject to a number of supraspinal controlling influences, with descending inhibition being crucial for the prevention of hyper-reflexia. Disruption to the descending inhibition, for example by a spinal cord lesion, will release the reflex arc from supraspinal control. ${ }^{16}{ }^{17}$
The mechanism appears to involve reciprocal Ia nerve inhibition, the level of which is related to the amount of spasticity and recovery of function in SCI. ${ }^{18}$ This disinhibition of spinal reflexes, resulting in hyper-reflexia or even spasticity, should therefore be useful as an indicator of the level of lesion and also as a tool for monitoring recovery from spinal cord injury. Spinal reflexes can be elicited by activating either the mechanoreceptors present in the interspinous ligaments, ${ }^{19}$ or by activating the muscle spindles in paraspinal muscles. ${ }^{15}$ We used a mechanical method to preferentially stimulate either mechanoreceptors (interspinous ligaments) or muscle spindles (in ES muscles) at different thoracic levels, and recorded short latency reflex responses in spinal cord injured and control subjects.

\section{METHODS}

\section{Subjects}

For the study, 11 healthy control individuals (three women, eight men; age range 24-46 years) were recruited. None had ever suffered from a neurological or psychological disorder and none was taking medication. The patient group consisted of 12 subjects (four women, eight men) with spinal cord injury. The level of lesions ranged from T2 to T12. All of these SCI subjects had sustained their injury at least 12 months prior to the investigation, and all were diagnosed with clinically complete lesions. None of the patients was on antispasticity medication at the time of investigation.

All subjects gave informed written consent. The study conformed to the Declaration of Helsinki, and ethical approval for the study was obtained from the ethics committees of Riverside Research and Stoke Mandeville Hospital NHS Trust.

Abbreviations: ASIA, American Spinal Injury Association; ES, erector spinae; $\mathrm{SCl}$, spinal cord injury 


\section{Test sites}

For the control subjects, reflexes were tested at neurological levels T2, T7, T11, and L4. For the SCI subjects, at least four sites were tested, one of which was at the level of lesion (according to ASIA assessment). The other sites included levels both above and below the level of lesion. For high (T1T6) thoracic levels, we chose two sites below and one above the lesion, and for low (T7-T12) thoracic lesions we chose two sites above and one below the level of lesion.

\section{Electromyographic recordings}

Surface EMG recordings were made from the right erector spinae (ES) muscles at the vertebral level of stimulation. Firstly, the thoracic and upper lumbar spinal processes were identified by palpation and by counting down from the more prominent seventh cervical process. The appropriate levels were marked with a felt tipped pen for easy identification and placement of EMG electrodes. Two self adhesive $\mathrm{Ag} / \mathrm{AgCl}$ surface EMG electrodes (Arbo Neonatal Blue; $2 \mathrm{~cm}$ diameter) were placed over the belly of the muscle (identified using palpation) at positions $3 \mathrm{~cm}$ and $6 \mathrm{~cm}$ lateral to the spinous process. Four pairs of electrodes were placed lateral to the four chosen spinous processes. EMGs were filtered $(-3 \mathrm{~dB}$ below $100 \mathrm{~Hz}$ and above $2 \mathrm{kHz})$ and amplified $(\times 10000)$ before being sampled $(4 \mathrm{kHz})$ by a computer for storage and analysis (Cambridge Electronic Design 1401; SIGNAL software; IBM compatible PC).

\section{Mechanical stimulation and protocol}

Subjects were seated comfortably in a low backed chair or wheelchair, with their arms resting on a table in front of them, allowing their back to remain supported and vertical. A blunt perspex probe with an end diameter of $5 \mathrm{~mm}$ was attached to an electromagnetic servo and could be angled to provide mechanical stimulation to a particular spinal process or over the midline of the ES muscle. Stimulation was applied over the spinous process (primarily to activate receptors in interspinous ligaments) or over the ES muscle (primarily to activate muscle spindles) just lateral to the process on the same side as the EMG recording. The electromagnetic servo applied the probe at a frequency of $\mathrm{l} \mathrm{Hz}$ and a throw of $3 \mathrm{~mm}$. Two trials of 50 mechanical prods were conducted at each of the eight probe sites; four on the spinous process and four on the right ES muscle. Electromyographic reflex responses in ES from the recording site adjacent to the prod (that is, at the same vertebral level) were full wave rectified and averaged with respect to the stimuli. To check the degree of mechanical spread of the stimulus, an accelerometer was attached to the adjacent muscle while the spinous process was prodded at the same spinal level. The movement (derived from the accelerometer signal) at the adjacent muscle was $<10 \%$ of that measured next to the probe; likewise, the movement at the spinous process was $<10 \%$ of that at the adjacent muscle when the muscle was prodded.

\section{Measurement and analysis}

In each of the two trials at each segmental level tested, the 50 EMG responses at the level of stimulation were full wave rectified and averaged. Presence or absence of short latency reflexes was noted in each trial. If necessary, a paired $t$ test comparing pre-stimulus EMG with that recorded at the time of the expected reflex was used to check for presence of a reflex, with $\mathrm{p}<0.05$ taken as statistically significant. Area and latency of the rectified average response was measured for each trial, and an average value noted for each segmental level tested in each subject. The incidence of a reflex at any given level was scored as either l (reflexes evident in both trials), 0.5 (reflexes present in only one of the two trials), or 0 (reflexes absent on both trials). Data from above the lesion, at the lesion level, and below the lesion were compared with controls using one way analysis of variance on ranks with Dunn's correction. Within the patient group, reflex sizes at and below the level of the lesion were examined to see whether they fell outside two standard deviations of the mean reflex size above the lesion.

\section{RESULTS}

\section{Raw data}

Fig 1 illustrates examples of averaged, rectified reflex responses elicited above, at the level of, and below the lesion in two spinal cord injured patients (both ASIA grade A complete). Panel A shows reflex responses from a patient with a clinical level of lesion level at $\mathrm{T} 4$ when the spinous processes were stimulated, and panel B shows reflex responses from a patient with a clinical level of lesion level at T7 when the adjacent muscle sites were stimulated.

\section{Incidence and latency of reflexes}

In control subjects, the overall incidence of a reflex (at all levels tested) was $81.8 \%$ in response to prodding the process and $79.5 \%$ in response to prodding the muscle. In general, the SCI subjects showed a greater incidence of reflex responses compared with controls when the process was prodded above or below the lesion $(80-100 \%)$ with a similar incidence $(80 \%)$ at the level of lesion (fig 2A). When the muscle was prodded (fig $2 \mathrm{C}$ ) reflexes were seen in almost every trial and were only absent in a few cases when tested at one vertebral level below the lesion.

The overall mean (SEM) latency of the response in controls (all four levels tested) was $5.72(0.53) \mathrm{ms}$ when prodding the spinous process and was $5.42(0.42) \mathrm{ms}$ while prodding the muscle. Slightly, but insignificantly $(\mathrm{p}>0.05)$, longer latency reflexes were recorded SCI patients in response to both types of stimuli at all levels. When the process was prodded (fig $2 \mathrm{~B}$ ) the latency of responses $2-5$ segments below the lesion $(6.34$ (0.3) ms) was no different ( $p>0.05)$ to responses at the level of the lesion $(6.04(0.1) \mathrm{ms})$ or 2-7 segments above the lesion $(6.00(0.25) \mathrm{ms})$. Likewise, when the muscle was prodded (fig 2D) the latency of responses below the lesion $(6.12$ (0.57) $\mathrm{ms})$ was no different $(\mathrm{p}>0.05)$ to responses at the level of the lesion (6.14 (0.17) ms) or above the lesion (5.93 (0.21) $\mathrm{ms}$ ).

\section{Size of the reflex responses}

Fig 3 compares the size of reflex responses to prodding the spinous process (A) or the muscle (B) as a percentage of an equivalent period of background EMG at different vertebral levels in relation to the spinal cord lesion. The levels tested are aligned with respect to the lesion level. For example, in a patient whose lesion level was T6 and the sites T2, T6, T8, and T10 were prodded, the reflex response from $\mathrm{T} 2$ is included in the white bar four segments above the lesion, the response from T6 in the black bar at the lesion level, the response from T8 in the grey bar two below the lesion and the response from T10 in the grey bar four below the lesion. The horizontal solid lines represent the mean reflex area obtained from the control subjects, while the dashed lines represent the standard deviation of this mean.

When the spinous process was prodded (fig 3A), mean reflex responses at the level of lesion and one segment either side of the lesion were larger than the mean size of all the responses recorded two or more levels above or below the lesion, but this difference was not statistically significant. We calculated the mean size of reflexes above the lesion and added two standard deviations of that mean to represent the $95 \%$ confidence limits. Six of the 11 patients showed reflexes around the level of lesion that exceeded $(\mathrm{p}<0.05)$ the $95 \%$ confidence interval (CI) of the responses above the lesion. 
A

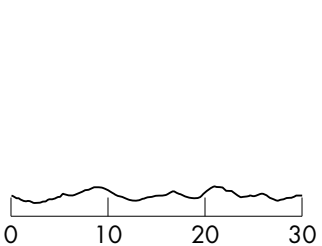

B Above lesion - T6

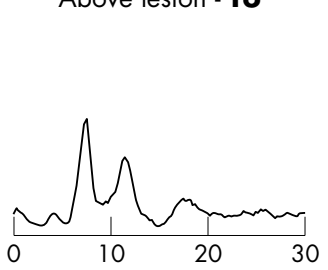

Above lesion - T2

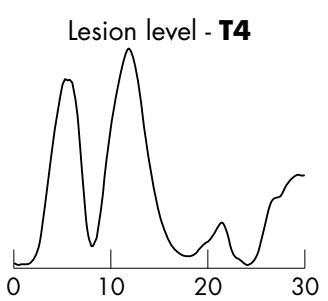

Lesion level - T7

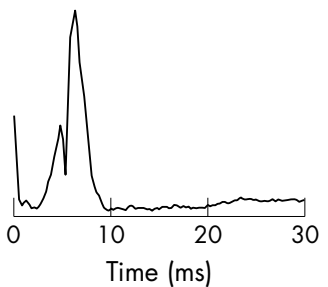

Below lesion - T6

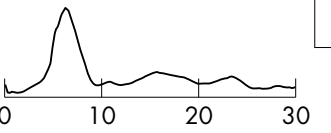

Below lesion - T9

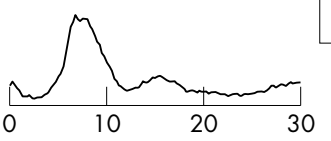

Figure 1 Reflex EMG responses to prodding a spinous process $(A)$ or the paraspinal muscle (B) in complete $\mathrm{SCl}$ patients. Each record represents an average of 50 rectified responses

$30 \mu \mathrm{V}$ recorded at the same vertebral level as the mechanical stimulus. The stimulus triggered the EMG recording at time zero. (A) Reflex responses evoked by prodding a spinous process from a patient with a clinical level of lesion at T4. (B) Reflex responses evoked by prodding the paraspinal muscle from a patient with a clinical level of lesion at T7. In both cases, the size of the response is clearly larger at the clinical level of the lesion than either above or $10 \mu \mathrm{V}$ below the lesion.
Overall, responses at the lesion level, and one segment either side $(268(60 \%)$ were significantly larger $(p<0.05)$ than the control responses (173 (18\%), but the pooled responses above (126 $(10 \%)$ or below (148 $(18 \%)$ the lesion were no different $(\mathrm{p}>0.05)$ to the controls.

There was a broadly similar result when the muscle was prodded (fig 3B), with hyper-reflexia evident at and around the lesion level. The mean reflex response was $588(226) \%$ at one segment above the lesion, and $566(282) \%$ at one segment below the lesion, but rather smaller (239 (75)\%), although still hyper-reflexive, at the clinical level of lesion. As for the process prodding, we calculated the mean size of reflexes above the lesion and added two standard deviations of that mean to represent the $95 \%$ confidence limits. Five of the 11 patients showed reflexes around the level of lesion that exceeded $(p<0.05)$ the $95 \%$ CI of the responses above the lesion. When compared with control subjects (148
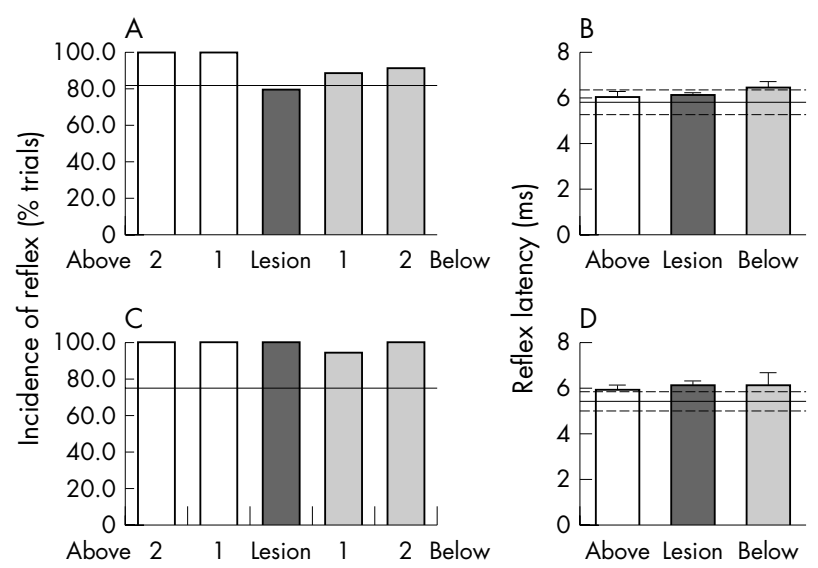

Figure 2 The incidence $(A, C)$ and latencies $(B, D)$ of reflex responses above, at, and below clinically complete spinal cord lesions. (A, B) Reflexes elicited by prodding the spinous process. (C, D) Reflexes elicited by prodding the muscle. In $A$ and $C$, white bars represent the incidence of responses one or two levels above the lesion, black bars represent responses at level of lesion and grey bars represent responses one or two levels below the lesion. In B and D, white bars represent latencies 2-7 segments above the lesion, black bars represent the lesion level plus one level either side, and grey bars represent 2-5 segments below the level of lesion. The solid lines represent the mean control latencies and the dashed lines the SEM.
(13)\%), the responses at and around the lesion (446 $(112) \%)$ were larger $(p<0.05)$, but there was no difference above (158 (26)\%) or below (299 (90)\%) the lesion level.

\section{Relationship of reflexes to background EMG in control subjects}

Figure 4 plots the size of the reflex response for each spinal level tested against the pre-prod EMG in each control subject while prodding spinous process (A) and the muscle (B). Only

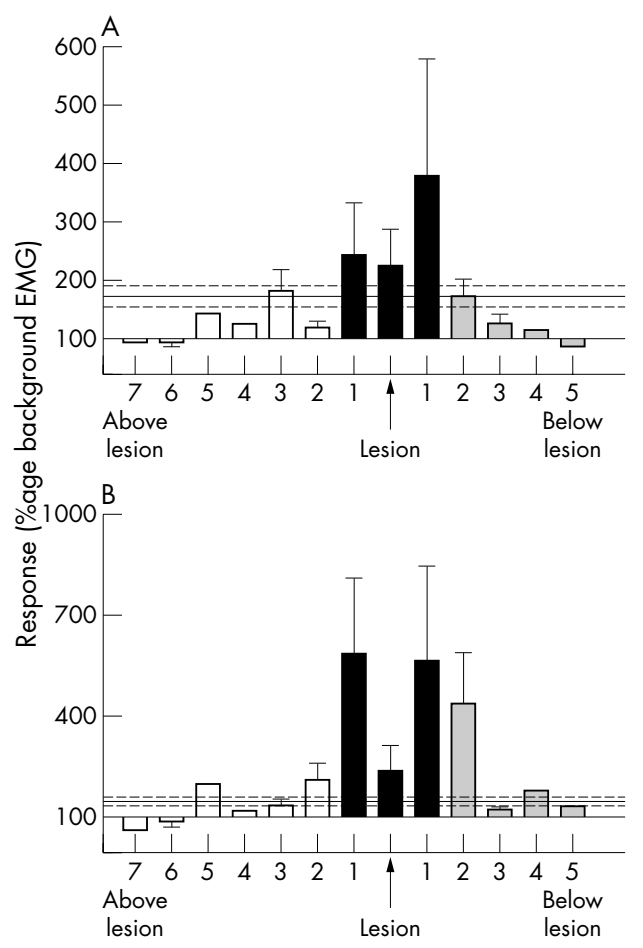

Figure 3 Size of reflex responses at vertebral levels relative to clinical level of lesion. Mean (SE) size of reflex responses as a percentage of the background EMG when prodding the spinous process (A) or the muscle (B) for the $\mathrm{SCl}$ subjects. Black bars show reflexes at or within one vertebral level of the lesion; open bars are the reflexes above the lesion; grey bars are the reflexes below the lesion. The mean size of reflexes evoked in control subjects is indicated by the solid line and dashed lines indicate SEM. 
while prodding the muscle at Tll was there a significant (linear regression analysis; $\mathrm{p}<0.05 ; \mathrm{r}^{2}=0.77$ ) positive correlation between reflex size and the level of pre-prod EMG. At all other spinal levels and at T11 while prodding the spinous process, there was no correlation $(\mathrm{p}>0.05)$ between the size of a reflex response and the background EMG. These data, taken alongside the results in fig 3, suggest that the hyperreflexia observed around the level of the lesion in SCI is not simply a consequence of increased resting tone in the muscle; the reflex area, as a percentage of background EMG, is far greater around the level of lesion than at other levels.

\section{DISCUSSION}

It has been shown that reflex control of paraspinal musculature is mediated by sensory receptors in the numerous viscoelastic spinal structures such as the intervertebral disc and the spinal ligaments. ${ }^{20}$ Short latency reflex activation of the paraspinal muscles can be achieved by stimulating the mechanoreceptors in the passive structures such as ligaments. ${ }^{19}$ Reflex activation may also be achieved by stimulating the muscle spindles, giving rise to a monosynaptic stretch reflex. Reflexes in the paraspinal muscles have

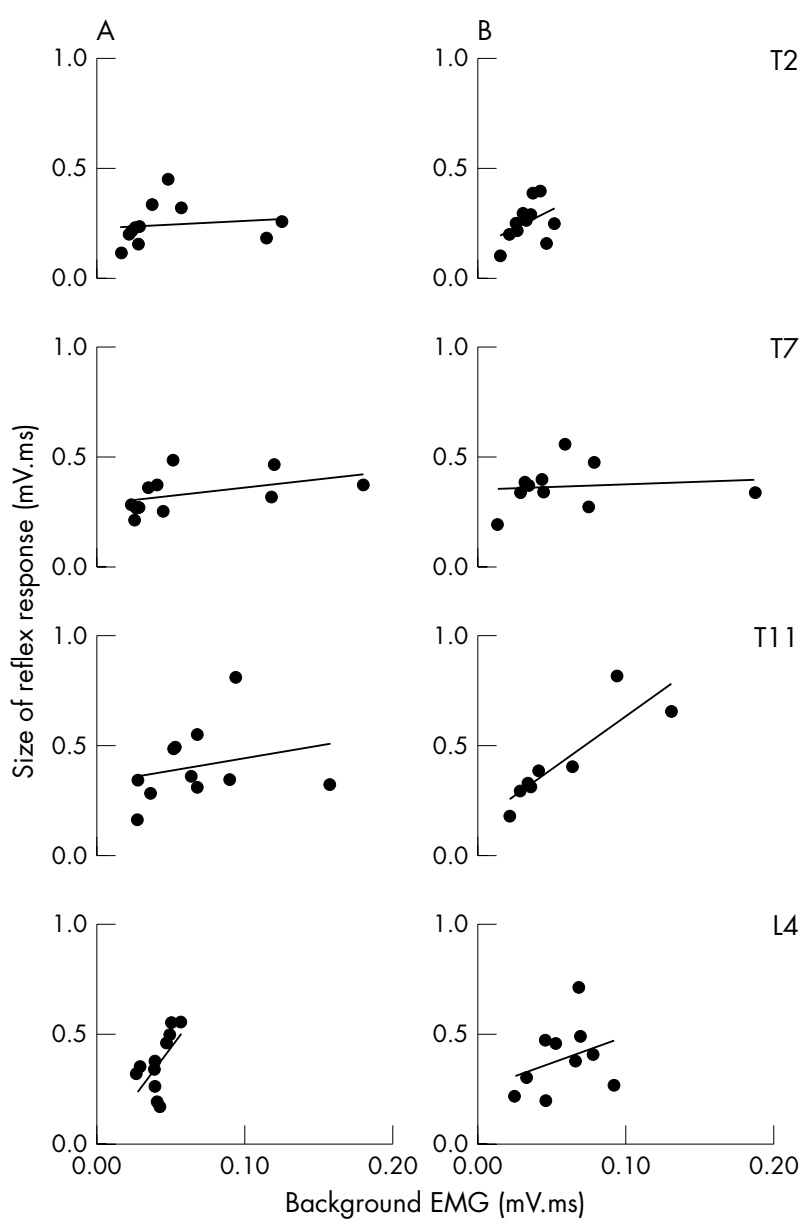

Figure 4 Correlations between pre-prod EMG and the size of the reflex response in paraspinal muscles when prodding $(A)$ the spinous process and (B) the muscle at four different spinal levels in control subjects. Each data point represents average values from two trials conducted at that prod site or from one trial if reflexes were evoked in only one of the two trials. If no reflexes were evoked at a particular level in an individual then no point is plotted. A positive correlation between reflex size and preprod EMG was observed only when prodding the muscle at T1 1 (linear regression; $\left.p<0.05 ; r^{2}=0.77\right)$. No correlation was seen at any other spinal level or when prodding the spinous process at T1 1. The solid lines are lines of linear regression. been studied extensively in the lumbar region, ${ }^{19}{ }^{20}$ because it has been thought that damaged spinal structures could activate the paraspinal muscles continuously, leading to muscle spasms giving rise to back pain. This hypothesis has been minimally explored in the thoracic levels. ${ }^{15}$ In the present study, we have documented the pattern of reflex activation in the paraspinal muscles by stimulating vertebral mechanoreceptors and muscle spindles in the thoracic region in a normal adult population and a group of spinal cord injured patients.

The stimuli applied in this study are not likely to be specific for either mechanoreceptors in the ligaments (spinous process prod) or muscle spindles (muscle prod). When mechanical stimulus spread was investigated using an accelerometer, it was found that movement at the muscle (to spinous process prod) or at the spinous process (to muscle prod) was only $10 \%$, or less, of that recorded at the site of prod. Thus, it is likely that the spinous process prod predominantly activates receptors in the intraspinous ligaments and that muscle prod predominantly activates muscle spindles.

\section{Hyper-reflexia around the level of lesion}

Exaggerated stretch reflexes in upper motor neurone disorders have been attributed to hyper-excitability of the motor neurone pool resulting from loss of supra-spinal inhibition. ${ }^{16}{ }^{17}$ As might be predicted therefore, hyperexcitability has been seen at and around the clinical level of a spinal cord lesion in this study (fig 3). However, while prodding the muscle, the hyper-reflexia was not so marked at the segment reflecting the clinical level of lesion compared with one segment above or below. This could be due to motor neurone loss together with reduced descending inhibition leaving the surviving motor neurones hyper-reflexive. There was considerable variability between SCI patients but, in general, reflex responses were similar to the controls when recorded three or more vertebral segments either above or below the level of the lesion.

\section{Background EMG}

It has been clearly established in previous studies that the level of background EMG has a direct relationship to the excitability of the motor neurones. ${ }^{21}{ }^{22}$ In agreement, there was a weak relationship between the size of the reflex and the amount of background EMG in the control population (fig 4). However, despite the influence of background EMG, the hyper-reflexia is independent of activity in the muscle. Even when the size of the reflexes was expressed as a percentage of the background EMG there is pronounced hyper-reflexia around the level of lesion (fig 3) that cannot be accounted for by the weak relationship observed in the control recordings (fig 4).

\section{Incidence of reflexes}

In general the incidence of reflexes in the patients was greater than in the controls (fig $2 \mathrm{~A}, \mathrm{C}$ ). Hyper-reflexia around the level of the lesion in the patients would enhance all responses, making otherwise small reflexes easier to identify; this might partly account for the higher incidence of reflexes. The only exception to the higher incidence of reflexes in SCI was at the clinical level of lesion when the spinous process was prodded (see fig 2). A possible cause for this might be damage to the mechanoreceptors in the spinal ligaments. Ligaments, being passive inelastic structures, are more susceptible to damage in the event of trauma. ${ }^{23}$

\section{Latency of reflex responses}

The latencies of the reflexes in spinal cord injury patients were not significantly different from control subjects and did 
not change significantly with respect to the level of lesion (fig 2). However, on average, the mean latency of response in SCI, at all levels, was slightly longer than in the controls and it is worth considering possible reasons for this. Assuming that there was no physical loss of motor neurones, any increase in latency could be due to an alteration in axonal conduction velocity as observed in other pathologies. ${ }^{24} 25$ Another consideration is that the erector spinae muscles are supplied by both fast and slow conducting nerve fibres, ${ }^{26}$ and the recruitment order of motor neurones is correlated with the conduction velocity. ${ }^{27}$ It has been shown that disuse of the muscles can lead to death of motor neurones, and that the first to succumb are the fast conducting neurones. ${ }^{26}$ The loss of fast conducting motor neurones below the level of lesion caused by disuse atrophy of the erector spinae muscles could account for the lengthening of latency below the level of lesion. However, countering this argument, studies investigating changes to peripheral nerves following spinal cord injury have found no change in conduction velocity ${ }^{28-30}$ The consistent but insignificant increase in reflex latency above the level of the lesion remains unexplained.

\section{Clinical use of the reflexes}

The distinct pattern of hyper-reflexia emerging from this study would be a useful tool to monitor the recovery and reinnervation after therapeutic intervention. Regeneration of descending axons following such intervention might reestablish descending inhibition and bring the reflexes back to normal level. Nevertheless, cautious interpretation is required, because at the level of lesion the damage to the inter-vertebral disc, joints and ligaments and loss of motor neurones cannot be reversed. As a result of possible damage to the mechanoreceptors in the interspinous ligaments, mild hyporeflexia can be expected after re-establishment of descending inhibition.

The erector spinae muscles receive a multi-segmental innervation, but nevertheless show distinct vertebral levels of hyper-reflexia in SCI. A previous study in our laboratory showed that motor evoked potentials in ES (to cortical stimulation) paradoxically appear to occur below the level of a clinically complete lesion, ${ }^{14}$ and this may be due to the multi-segmental innervation of paravertebral muscles from above the lesion. Although appearing to negate the usefulness of cortically evoked responses in determining the level of SCI, a pattern of increasing cortical thresholds to transcranial magnetic stimulation was observed related to the level of injury. The short latency reflexes examined in this study show a more definite pattern of change around the clinical level of the lesion, despite this multi-segmental innervation.

\section{CONCLUSION}

This study provides evidence that mechanically evoked segmental reflexes in back muscles can be used as a diagnostic tool to help identify the level of complete thoracic spinal cord lesions. In the future, such techniques may prove invaluable for serial monitoring of the status of spinal cord lesions in patients following interventional therapies to repair the spinal cord.

\section{Authors' affiliations}

A Kuppuswamy, S Theodorou, M Catley, P H Ellaway, N J Davey, Division of Neuroscience and Psychological Medicine, Imperial College Faculty of Medicine, Charing Cross Campus, London W6 8RP, UK P H Strutton, A H McGregor, Department of Orthopaedic and Trauma Surgery, Imperial College Faculty of Medicine, Charing Cross Campus, London W6 8RP, UK
A Kuppuswamy, S Theodorou, M Catley, P H Ellaway, N J Davey, The National Spinal Injuries Centre, Stoke Mandeville Hospital, Aylesbury, Bucks HP21 8AL, UK

Competing interests: none declared

\section{REFERENCES}

1 Cohen ME, Ditunno JF Jr. Donovan WH, et al. A test of the 1992 International Standards for Neurological and Functional Classification of Spinal Cord Injury. Spinal Cord 1998;36:554-60.

2 Kirshblum SC, Memmo P, Kim N, et al. Comparison of the revised 2000 American Spinal Injury Association classification standards with the 1996 guidelines. Am J Phys Med Rehabil 2002;81:502-5.

3 Curt A, Schwab ME, Dietz V. Providing the clinical basis for new interventional therapies: refined diagnosis and assessment of recovery after spinal cord injury. Spinal Cord 2004;42:1-6.

4 Ellaway PH, Anand P, Bergstrom EM, et al. Towards improved clinical and physiological assessments of recovery in spinal cord injury: a clinical initiative. Spinal Cord 2004;42:325-3.

5 Blesch A, Tuszynski MH. Cellular GDNF delivery promotes growth of motor and dorsal column sensory axons after partial and complete spinal cord transections and induces remyelination. J Comp Neurol 2003;467:403-17.

6 Keyvan-Fouladi N, Raisman G, Li Y. Functional repair of the corticospinal tract by delayed transplantation of olfactory ensheathing cells in adult rats. J Neurosci 2003;23:9428-34.

7 Poynton AR, O'Farrell DA, Shannon F, et al. An evaluation of the factors affecting neurological recovery following spinal cord injury. Injury 1997;28:545-8.

8 Curt A, Rodic B, Schurch B, et al. Recovery of bladder function in patients with acute spinal cord injury: significance of ASIA scores and somatosensory evoked potentials. Spinal Cord 1997;35:368-73

9 Curt A, Nitsche B, Rodic B, et al. Assessment of autonomic dysreflexia in patients with spinal cord injury. J Neurol Neurosurg Psychiatry 1997;62:473-7.

10 Curt A, Dietz V. Ambulatory capacity in spinal cord injury: significance of somatosensory evoked potentials and ASIA protocol in predicting outcome. Arch Phys Med Rehabil 1997;78:39-43.

11 Curt A, Keck ME, Dietz V. Functional outcome following spinal cord injury: significance of motor-evoked potentials and ASIA scores. Arch Phys Med Rehabil 1998:79:81-6.

12 Hashimoto T, Uozumi T, Tsuji S. Paraspinal motor evoked potentials by magnetic stimulation of the motor cortex. Neurology 2000;55:885-8.

13 Smith HC, Savic G, Frankel HL, et al. Corticospinal function studied over time following incomplete spinal cord injury. Spinal Cord 2000;38:292-300.

14 Cariga P, Catley M, Nowicky AV, et al. Segmental recording of cortical motor evoked potentials from thoracic paravertebral myotomes in complete spinal cord injury. Spine 2002;27:1438-43.

15 Tani T, Yamamoto H, Ichimiya M, et al. Reflexes evoked in human erector spinae muscles by tapping during voluntary activity. Electroencephalogr Clin Neurophysiol 1997; 105:194-200.

16 Mayer NH. Clinicophysiologic concepts of spasticity and motor dysfunction in adults with an upper motoneuron lesion. Muscle Nerve Suppl 1997;6:S1-13.

17 Sheean G. The pathophysiology of spasticity. Eur J Neurol 2002;9(suppl 1):3-9.

18 Okuma Y, Mizuno Y, Lee RG. Reciprocal la inhibition in patients with asymmetric spinal spasticity. Clin Neurophysiol 2002;113:292-7.

19 Solomonow $M$, Zhou BH, Harris $M$, et al. The ligamento-muscular stabilizing system of the spine. Spine 1998;23:2552-62.

20 Holm S, Indahl A, Solomonow M. Sensorimotor control of the spine. J Electromyogr Kinesiol 2002;12:219-34.

21 Nakazawa K, Yamamoto SI, Yano H. Short- and long-latency reflex responses during different motor tasks in elbow flexor muscles. Exp Brain Res 1997; 1 16:20-8.

22 Ogiso K, McBride JM, Finni T, et al. Short-latency stretch reflex modulation in response to varying soleus muscle activities. J Electromyogr Kinesiol 2002;12:17-26.

23 Solomonow M, Zhou BH, Baratta RV, et al. Biomechanics of increased exposure to lumbar injury caused by cyclic loading: Part 1. Loss of reflexive muscular stabilization. Spine 1999;24:2426-34.

24 Emeryk-Szajewska B, Badurska B, Kostera-Pruszczyk A. Electrophysiological findings in hereditary motor and sensory neuropathy type I and IIconduction velocity study. Electromyogr Clin Neurophysiol 1998;38:95-101.

25 Andersen H, Stalberg E, FalckB. F-wave latency, the most sensitive nerve conduction parameter in patients with diabetes mellitus. Muscle Nerve 1997;20:1296-302.

26 Kalimo H, Rantanen J, Vilianen T, et al. Lumbar muscles: structure and function. Ann Med 1989;21:353-9.

27 Bawa P, Binder MD, Ruenzel P, et al. Recruitment order of motoneurons in stretch reflexes is highly correlated with their axonal conduction velocity. J Neurophysiol 1984;52:410-20.

28 Brouwer B, Bugaresti J, Ashby P. Changes in corticospinal facilitation of lower limb spinal motor neurons after spinal cord lesions. J Neurol Neurosurg Psychiatry 1992;55:20-4.

29 Curt A, Keck ME, Dietz V. Clinical value of F-wave recordings in traumatic cervical spinal cord injury. Electroencephalogr Clin Neurophysiol 1997; 105: 189-93.

30 Tsai CT, Chen HW, Chang CW. Assessments of chronodispersion and tacheodispersion of $\mathrm{F}$ waves in patients with spinal cord injury. Am J Phys Med Rehabil 2003;82:498-503. 